\title{
Usefulness of Neuronavigation During Cranial Surgery.
}

\author{
Pankaj Raj Nepal $^{1}$, Suman Rijal ${ }^{1}$ \\ ${ }^{1}$ Department of Neurosurgery, Upendra Devkota Memorial National Institute of \\ Neurological and Allied Sciences, Bansbari, Kathmandu, Nepal

\section{Correspondence:} \\ Dr. Pankaj Raj Nepal \\ Head of Department, Department of Neurosurgery, B \& C Medical College and \\ Teaching Hospital \& Research Center,Birtamode,Jhapa,Nepal. \\ Email: pankajrajnepal@gmail.com \\ Phone: +9779801098106
}

Background: Neuronavigation helps neurosurgeons by providing intraoperative three dimensional (3D) view of CT/MRI of the patients thus helping the surgeon in different steps of surgery. With the objective to evaluate the usefulness of neuronavigation in early years after installment, this study was conducted at a tertiary care center in Nepal. Methodology: This is a prospective analytical study, where all the consecutive cases of the neurosurgical cranial cases using frameless stereotactic (Medtronics stealth station 7), were considered during May 2014 to March 2017. All the lesions were categorized according to the location and were evaluated using the Image guided surgery (IGS) score. Analysis was done using stratification and percentage system.

Result: There were total 122 cases operated using neuronavigation during the study period, with ages ranged between 5 to 83 years with mean age 38.32(SD16.09) years. $57.4 \%$ were male and $42.6 \%$ were female. Overall IGS scoring of the neuronavigation in the different location showed irreplaceability in $100 \%$ cases of convexity, ganglionic/ deep seated/ ventricle and brain stem lesion; however, it was more useful than conventional methods in $70.37 \%$ of lobar cases. Similarly, overall IGS scoring of neuronavigation in the different type of lesions showed irreplaceable in $80 \%$ of meningiomas, and $41.17 \%$ of gliomas; however, was more useful than conventional methods in $56.86 \%$ of gliomas

Conclusion:Use of neuronavigation seems to be irreplaceable for planning and raising flaps, in cases of convexity lesions and most of the cases of lobar and ganglionic/ deep seated leasions.

Key Words: Neuronavigation, Frameless stereotactic, Image guided surgery, Meningioma, Steriotatic biopsy.

$\mathrm{N}$ euronavigation has been commonly used in neurosurgical theatre since last several years throughout the world; however, in the developing country its use is limited. ${ }^{1}$ This limitation is mainly due to high cost involved in the installation. ${ }^{1}$

13

Date Submitted: $11 / 8 / 2019$

Date Accepted: 12/9/2019
Neuronavigation helps neurosurgeons by providing intraoperative $3 \mathrm{D}$ view of CT/MRI of the patients thus helping the surgeon during flap marking, placement of burr hole, delineation of anatomy, planning the route of surgical procedure or biopsy

egneuro, Volume 01, Issue 02, 2019

DOI: https://doi.org/10.3126/egn.v1i2.25729 
procedures and even in the advanced robotic neurosurgery. ${ }^{2-4}$ Neuronavigation also supports several sequences of MRI like, angiography and tractography, thus anatomical deliniation is better obtained even with vascular lesion like AVM. 5,6

With the objective to evaluate the usefulness of neuronavitiaon in early years of instalment, this study was conducted at a tertiary care center in Nepal.

\section{Methodology:}

This is a prospective analytical study, where all the consecutive cases of the neurosugical cranial cases using frameless steriotatic (Medtronics stealth station 7), from May 2014 to March 2017 were considered.

For planning of neuronavigation guided surgery, $1 \mathrm{~mm}$ thin sliced CT/ MRI were obtained, which include the tip of nose to the vertex of the respected patient. For cases of vascular lesions like AVM, similar MRI with MRA were obtained. These images were installed in the Medtronics stealth station 7, then the lesion was mapped. Patient head was fixed in 3 pin head rest and the registration was done using patient's surface marking, except for posterior fossa lesions where fudicial markers were used in prone position.

Passive planner probe was used during designing of flap, placement of burr, providing the accurate trajectory to the lesion, and to see the extent of resection done.

All the lesions were categorized according to the location as lobar, convexity, ganglionic/ deep seated/ ventricular, sellar/ supra sellar, middle cranial fossa, posterior fossa and brain stem: and the usefulness of neuronavigation was evaluated using the Image Guidance Surgery (IGS) score (Table 1). ${ }^{2}$ Similarly, the types of lesions were also evaluated using the IGS. Analysis was done using stratification and percentage.

Table 1: Image- guided surgery scoring

\begin{tabular}{|l|l|}
\hline 1 a: & $\begin{array}{l}\text { Grading scale of utility of image- } \\
\text { guided system }\end{array}$ \\
\hline $0:$ & IGS not helpful or can be detrimental \\
\hline $1+:$ & $\begin{array}{l}\text { IGS helpful but procedure can } \\
\text { proceed without it }\end{array}$ \\
\hline $2+:$ & $\begin{array}{l}\text { IGS more useful than conventional } \\
\text { methods }\end{array}$ \\
\hline $3+:$ & IGS irreplaceable \\
\hline 1 b: & $\begin{array}{l}\text { Cranial IGS score } \\
\text { a) Design of flap/ burr hole+ } \\
\text { b) Delineation of anatomy + } \\
\text { c) Navigation/ access to lesion+ } \\
\text { d) Resection/ biopsy/ procedure }\end{array}$ \\
\hline $0-3:$ & $\begin{array}{l}\text { IGS not helpful but procedure can be } \\
\text { continued without it }\end{array}$ \\
\hline $4-6:$ & $\begin{array}{l}\text { IGS helpful but procedure can be } \\
\text { continued without it }\end{array}$ \\
\hline $7-9:$ & $\begin{array}{l}\text { IGS more useful than conventional } \\
\text { methods }\end{array}$ \\
\hline $10-$ & $\begin{array}{l}\text { IGS irreplaceable } \\
12:\end{array}$ \\
\hline
\end{tabular}

IGS: Image- guided surgery

\section{Result:}

There were total 122 cases operated using neuronavigation during the study period, with ages ranged between 5 to 83 years where mean age was 38.32 (SD 16.09) years. There were $57.4 \%$ male and $42.6 \%$ female.

Of all the 122 cases operated using neuronavigation, $41.8 \%$ were glioma, $28.68 \%$ were meningioma, $9 \%$ were pituitary macroadenomas, $6.56 \%$ were AVMs and rest of them were of different pathologies ( Figure: 1). Most common location of lesion operated was for lobar (

egneuro, Volume 01,Issue 02, 2019 
$44.26 \%$ ), followed by convexity (23.77\%) and other different locations (Figure 2).

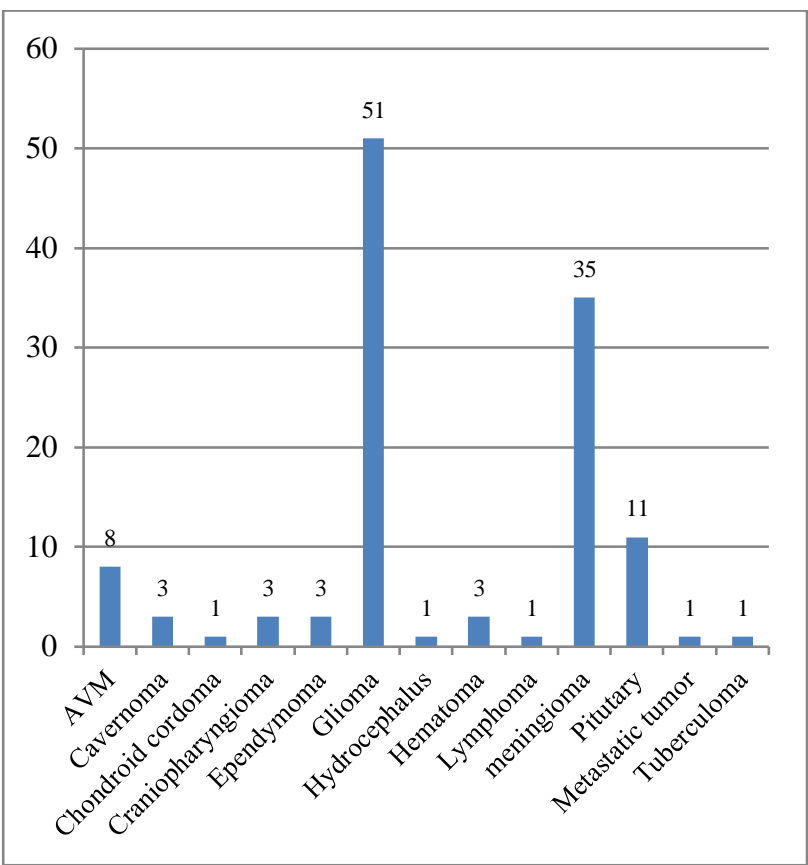

Figure 1: Case distribution by type of lesion.

The first category of IGS, which is flap design/ placement of burr has IGS 3 in $100 \%$ of lobar, and $100 \%$ of convexity lesions (Figure 3).

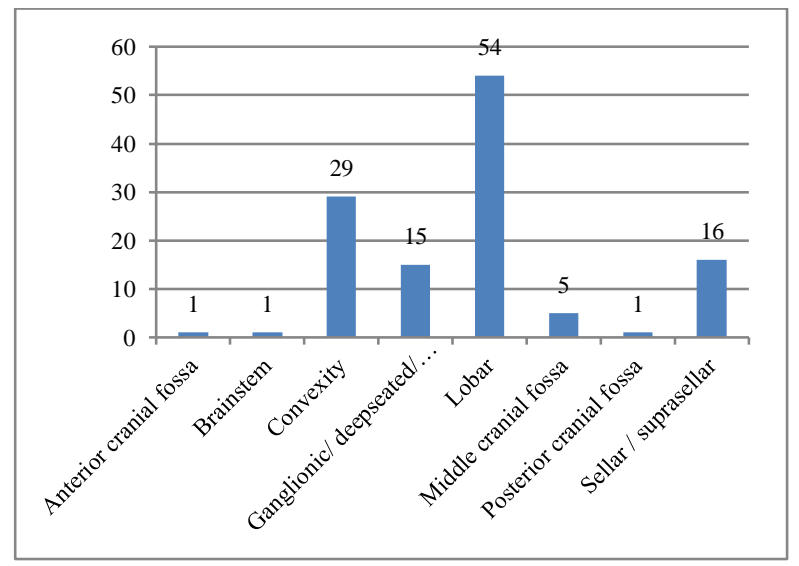

Figure 2: Total cases where neuronavigation was used by location.

egneuro, Volume 01, 2019

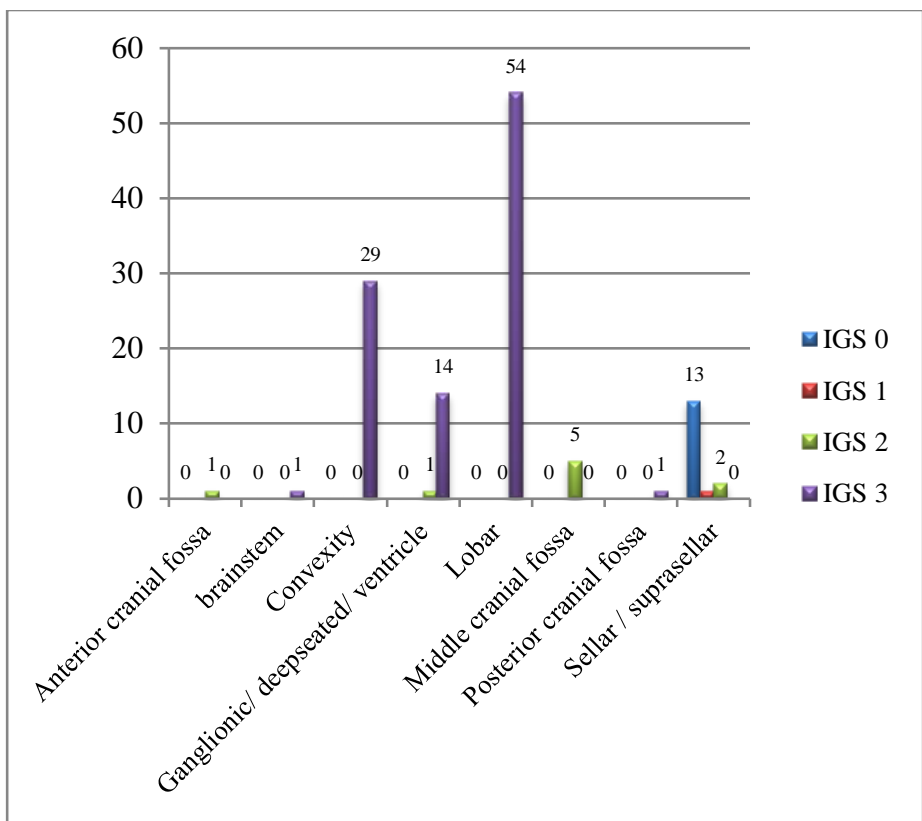

Figure 3: IGS score in flap design and placement of burr.

Similarly, for delineation of anatomy, IGS 3 was obtained in $70.37 \%$ of lobar, and $96.55 \%$ of convexity lesions (Figure 4). Anatomical delineation was best appreciated in cases of vascular lesions like AVM and cavernomas.

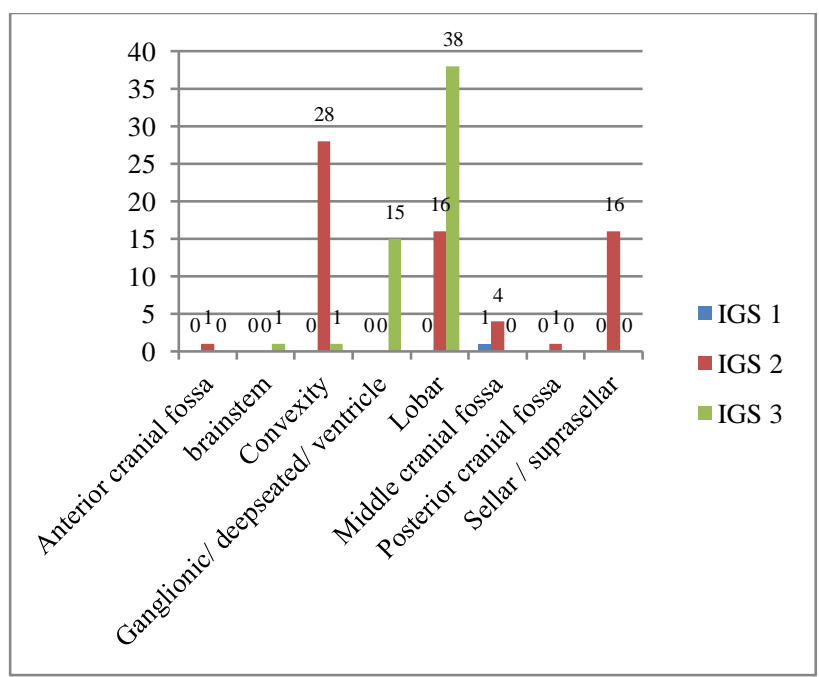

Figure 4: IGS score in delineation of anatomy.

For gaining access to the lesion, most of the lobar lesions (70.37\%) had IGS 2; however, 
IGS 3 was best seen in cases of convexity (100\%), ganglionic/ deep seated/ ventricle $(100 \%)$, and sellar/ suprasellar $(81.25 \%)$ lesions (Figure 5).

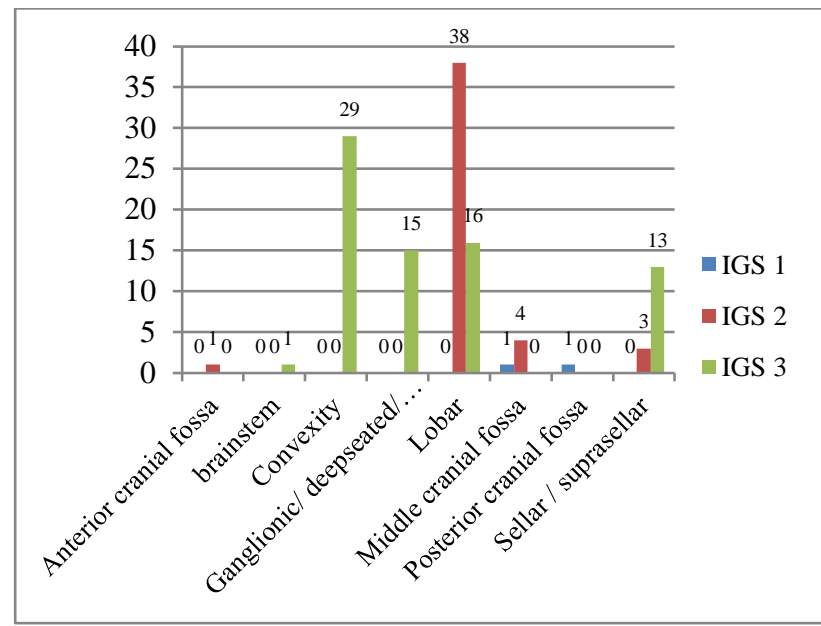

Figure 5: IGS score in gaining access

Use of neuronavigaion for completion of the procedure/ resection was not useful compared to the conventional technique in most of the case, except for convexity lesions where IGS 2 were seen in $96.55 \%$ of cases and was also useful in cases in shunt placement, and biopsy procedure ( Figure 6).

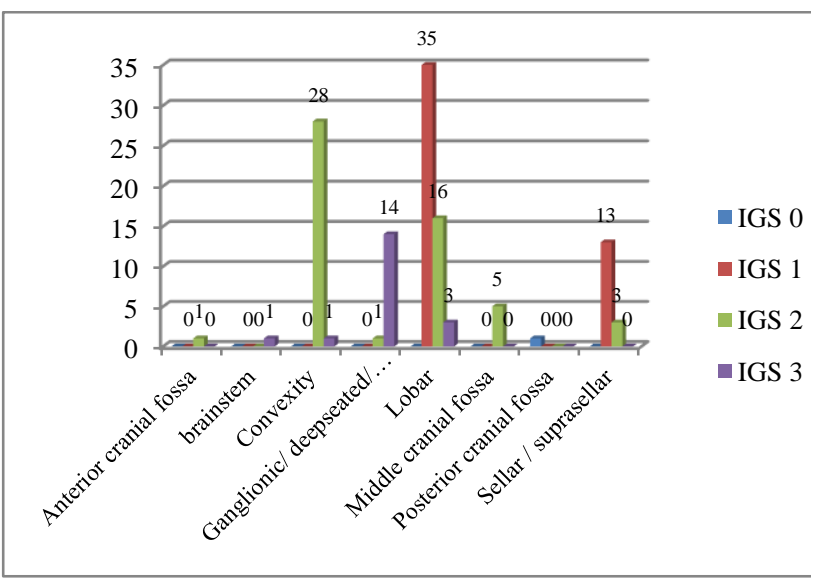

Figure 6: IGS score in resection/ biopsy/ procedure.

Overall IGS scoring of the neuronavigation in the different location was irreplaceable in 16
$100 \%$ cases of convexity, ganglionic/ deep seated/ ventricle and brain stem lesions; however, it was more useful than conventional methods in $70.37 \%$ of lobar cases (Figure 7). Similarly, overall IGS scoring of neuronavigation in the different type of lesions showed irreplaceable in $80 \%$ of meningiomas, and $41.17 \%$ of gliomas; however, was more useful than conventional methods in $56.86 \%$ of gliomas (Figure 8)

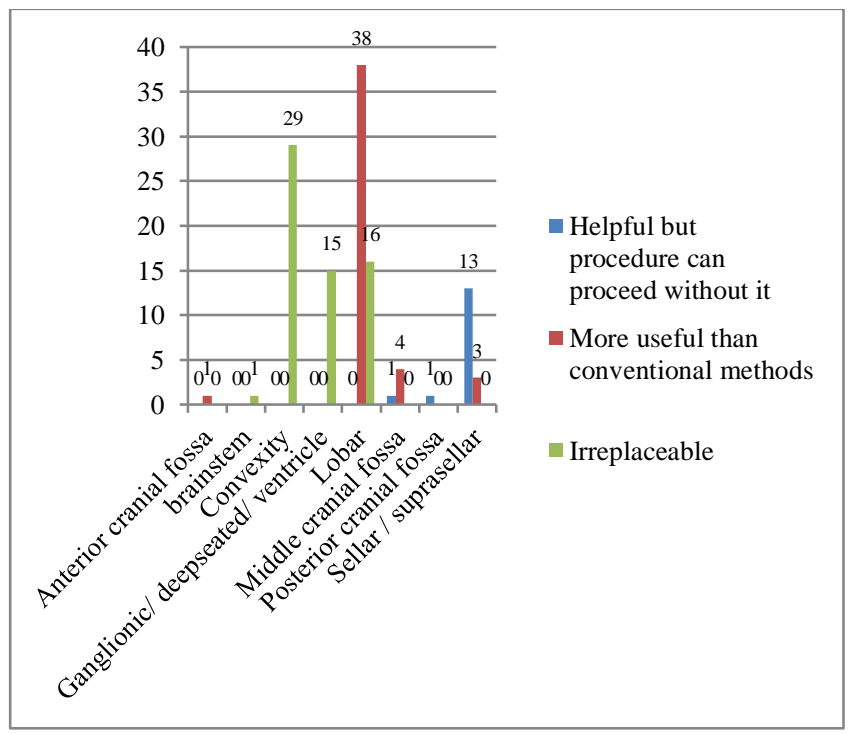

Figure 7: Overall neuronavigation use in different location.

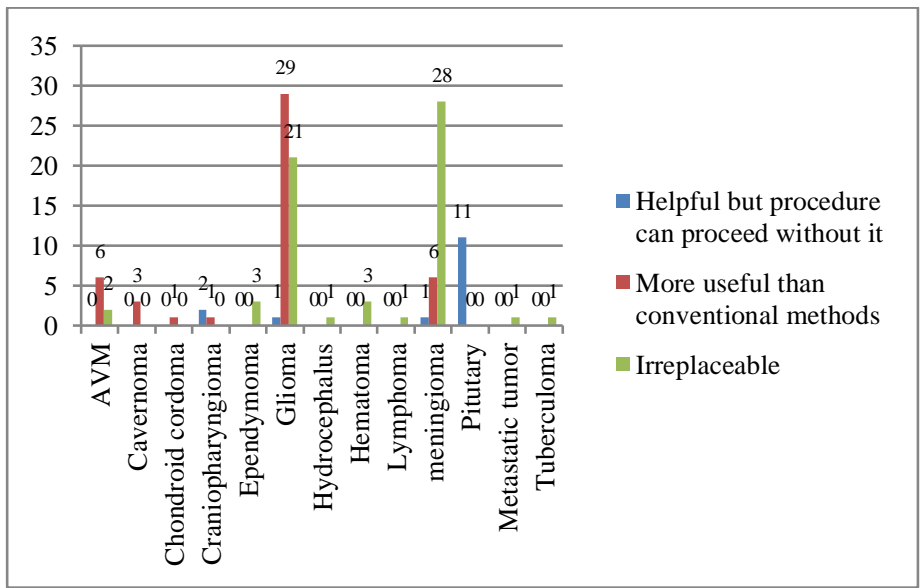

Figure 8: Overall neuronavigation use in different type of lesion.

egneuro, Volume 01,Issue 02, 2019 


\section{Discussion:}

Neuronavigation has made the neurosurgical practice safer than the conventional methods in terms of identification of critical anatomical structures intraoperatively and planning a safer approach during surgery. ${ }^{7,8}$ It has also reduced the size of craniectomies during a lot of neurosurgical procedures especially with those lesions located in the convexities. ${ }^{1,8}$

The use of neuronavigations in cranial cases doesn't seem to get restricted with the extreme of ages. Its use in children as small as 2 years has also shown a lot of benefit during neurosurgery. ${ }^{9-11}$ Similarly, in our study we have used neuronavigation in age ranged 5 to 83 years of age.

Among brain tumors glioma is the most common pathology encountered during neurosurgical practice followed by meningioma and pitutary adenomas. The similar distribution of disease was noted in our series for tumors where neuronavigation was used. Though the use of neuronavigation in most of the cases of meningiomas were irreplaceable, it was more useful than conventional methods and irreplaceable also in most of the cases of glioma. In cases of pituitary adenoma the procedure can proceed without neuronavigation even if it is helpful. When the lesions were categorized by location the most common location was at the lobar region, this distribution was mainly due to the higher percentage of the glioma in the series.

In cases of vascular lesions like AVM and cavernomas, neuronavigation was useful than the conventional methods. The MRA and MRV images when merged with the neuronavigation protocol MRI image, showed the best navigating means intraopratively for localization of feeding arteries, draining veins and the nidus; though one should be extremly careful while interpretating the image, which is done by comparing the MRA and MRV with the preoperative digital substraction angiography. The much better means of identifying the AVMs feeders, nidus and draining veins is by using the 3D doppler ultrasonography intraoperatively combined with the neuronavigating system. ${ }^{12} 13$

Neuronavigaton for lobar lesions was found irreplaceable for flap design/ placement of burr and most of them were also irreplaceable for delineation of anatomy. However, for the completion of the resection procedure most of them could be done without its use, this problem was mainly due to the brain shift and the navigating system was mainly based on the preoperative imaging system. These days there are lot of advancement in its resection procedures especially with the use of intraoperative MRI, and 3D ultrasonography intraoperatively combined with the navigation system. ${ }^{14,15}$ Use of 5ALA had also showed much better resection accuracy. ${ }^{16-18}$

The major problem during surgery with convexity lesion is the proper placement of the craniotomy flap for complete resection of the lesion, the brain shift usually doesn't possess a major problem, so use of neuronavigation has shown a major benefit. ${ }^{8}$, ${ }^{19}$ The benefit was mainly for localization of lesion and decreasing the size of the craniotomy ${ }^{8,}{ }^{19}$ Similarly, in our series, the use of neuronavigation for the convexity lesions were also irreplaceable for the flap design/ and placement of burr, and gaining access. However, most of them were useful than conventional methods for delineation of anatomy and completion of the resection procedure. 
Use of neuronavigaion in cases of sellar / parasellar lesions, also has shown benefit especially if the procedures were performed via the transsphenoidal route. ${ }^{20}$ For the trans- cranial approach to these areas, majority of the neurosurgeons seems to prefer to rely on the anatomical landmarks rather than the neuronavigations. In our series, in majority of cases use of neuronavigation was irreplaciable while gaining the access to the sella, and these were the cases where trans-sphenoidal route was done. The use of neuronavigation for delineation of anatomy by identifying the important structures was not helpful in our series.

Neuronavigation was irreplacable for planning and elevating the flap and gaining the access in all the cases of ganglionic/ deep seated and ventricular lesions, where

\section{References:}

1. Dwarakanath S, Suri A, Sharma BS, et al. Neuronavigation in a developing country: A pilot study of efficacy and limitations in intracranial surgery. Neurol India. 2007;55:111-6.

2. Golfinos JG, Fitzpatrick BC, Smith LR, et al. Clinical use of a frameless stereotactic arm: Results of 325 cases. J Neurosurg. 1995;83:197205.

3. Karas CS, Chiocca EA. Neurosurgical robotics: a review of brain and spine applications. Journal of Robotic Surgery. 2007;1(1):39-43.

4. Sutherland GR, Maddahi Y, Gan LS, et al. Robotics in the neurosurgical treatment of glioma. Surgical Neurology International. 2015;6(Suppl 1):S1-S8. the procedures like biopsy, evacuation of ganglionic hematoma and placement of ventriculo- peritoneal shunts were done. Similar benefit were also seen in different literatures, even in cases like placement of catheters in the slit ventricles. ${ }^{10}$ The biopsy procedures using neuronavigation are well described in the literature, with diagnostic yield of 87 to $97.4 \% .^{21-23}$ The errors in calculation in the deep seated ganglionic lesions were usually due to brain shift. ${ }^{24}$

Conclusion: The discovery of neuronavigation has definitely helped neurosurgeon in various prospects. Its use seems to be irreplaceable for planning and raising flaps in cases of convexity lesions and most of the cases of lobar and ganglionic/ deep seated lesions.

5. McGrath BM, Maloney WJ, Wolfsberger S, et al. Carotid artery visualization during anterior skull base surgery: a novel protocol for neuronavigation.

Pituitary. 2010;13(3):215-22.

6. Kamada K, Todo T, Masutani Y, et al. Combined use of tractographyintegrated functional neuronavigation and direct fiber stimulation. J Neurosurg. 2005 102(4):664-72.

7. Stadie AT, Kockro RA, Serra L, et al. Neurosurgical craniotomy localization using a virtual reality planning system versus intraoperative image-guided navigation. Int $\mathbf{J}$ Comput Assist Radiol Surg. 2011;6(5):565-72.

8. Jr EBS, Aurich LA, Jr. LFMS, et al. Neuronavigation for Intracranial Meningiomas. J Bras Neurocirurg. 2011;22 (4):143-9.

egneuro, Volume 01,Issue 02, 2019 
9. Walkden JS, Zador Z, Herwadkar A, et al. Use of intraoperative Doppler ultrasound with neuronavigation to guide arteriovenous malformation resection: a pediatric case series. Journal of Neurosurgery: Pediatrics. 2015;15(3):291-300.

10. Clark S, Sangra M, Hayhurst C, et al. The use of noninvasive electromagnetic neuronavigation for slit ventricle syndrome and complex hydrocephalus in a pediatric population: Clinical article. Journal of Neurosurgery: Pediatrics. 2008;2(6):430-4.

11. Roth J, Biyani N, Beni-Adani L, et al. Real-Time Neuronavigation with High-Quality 3D Ultrasound SonoWand ${ }$ in Pediatric Neurosurgery. Pediatric neurosurgery. 2007;43(3):185-91.

12. Mathiesen T, Peredo I, Edner G, et al. Neuronavigation for arteriovenous malformation surgery by intraoperative three-dimensional ultrasound angiography. Neurosurgery. 2007 60(4):345-51.

13. Unsgaard G, Ommedal S, Rygh OM, et al. Operation of arteriovenous malformations assisted by stereoscopic navigation-controlled display of preoperative magnetic resonance angiography and intraoperative ultrasound angiography. Neurosurgery. 2005 56(4):281-90.

14. Unsgaard G, Selbekk T, Müller TB, et al. Ability of navigated 3D ultrasound to delineate gliomas and metastases-comparison of image interpretations with histopathology. Acta neurochirurgica. 2005 147(12):1259-69.
15. Unsgaard G, Rygh OM, Selbekk T, et al. Intra-operative 3D ultrasound in neurosurgery. Acta neurochirurgica. 2006 148(3):23553.

16. Kaneko S, Kaneko S, 27;. J. Fluorescence-guided resection of malignant glioma with 5-ALA. International Journal of Biomedical Imaging. 2016.

17. Eljamel S. 5-ALA fluorescence image guided resection of glioblastoma multiforme: a metaanalysis of the literature. International journal of molecular sciences. 2015;16(5):10443-56.

18. Stummer W, Pichlmeier U, Meinel T, et al. ALA-Glioma Study Group. Fluorescence-guided surgery with 5aminolevulinic acid for resection of malignant glioma: a randomised controlled multicentre phase III trial. The lancet oncology. 2006;7(5):392401.

19. Awadalla AM, Khan A, Zaiton F. Management of High Convexity, Parasagittal and Falcine Meningiomas. Egyptian Journal of Neurosurgery. 2014;29 (2):25-36.

20. Hardy J. Neuronavigation in pituitary surgery. Surgical Neurology International. 1999;52(6):648-9.

21. Jain D, Sharma MC, Sarkar C, et al. Comparative analysis of diagnostic accuracy of different brain biopsy procedures. Neurology India. 2006;54(4):394.

22. Lobão CA, Nogueira J, Souto AA, et al. Cerebral biopsy: comparison between frame-based stereotaxy and neuronavigation in an oncology 
center. Arquivos de neuropsiquiatria. 2009;67(3B):876-81.

23. Nishihara M, Takeda N, Harada T, et al. Diagnostic yield and morbidity by neuronavigation-guided frameless stereotactic biopsy using magnetic resonance imaging and by framebased computed tomography-guided stereotactic biopsy. Surgical neurology international. 2014;5(Suppl 8):S421.

24. Coenen VA, Krings T, Weidemann $\mathrm{J}$, et al. Sequential visualization of brain and fiber tract deformation during intracranial surgery with three-dimensional ultrasound: an approach to evaluate the effect of brain shift. Operative Neurosurgery. 2005 56(1):133-41. 